\title{
Scanning tunneling microscopy on rough surfaces: Tip-shape-limited resolution
}

\author{
G. Reiss, J. Vancea, H. Wittmann, \&. Zweck, and H. Hoffmann \\ Institut für Angewandte Physik III, Universität Regensburg, Federal Republic of Germany 92002
}

(Received 29 June 1989; accepted for publication 26 September 1989)

This paper discusses the reliability of scanning tunneling microscopy (STM) images of mesoscopically rough surfaces. The specific structure of these images represents a convolution between the real surface topography and the shape of the tip. In order to interpret these images quantitatively, the line scans of steep and high steps can be used to obtain an image of the tip itself. This image shows tip radii ranging typically from 5 to $15 \mathrm{~nm}$ and cone angles of about $30^{\circ}$ over a length of $80 \mathrm{~nm}$, and can in turn be used to recognize the limits of STM resolution on a rough surface: High-resolution transmission electron microscopy cross-section images of $\mathrm{AL}$ island films on an $\mathrm{Au}-\mathrm{Nb}$ double layer are convoluted with the experimentally observed tip shape; the resulting line scans correspond very weil with STM graphs of the same samples. Finally ar overall criterion for the resolution of the STM on such surfaces is proposed.

\section{INTRODUCTION}

The infuence of the specific arrangement of the front atoms of a tip used for scanning tunneling microscopy (STM) has been intensively discussed in the literature. ${ }^{i-5}$ This arrangement, however, determines the resolution only in the case of atomically flat surfaces, showing features remarkably smaller than about $1 \mathrm{~nm}$. Such surfaces may be called microscopically rough and the resolution will be tip limited. In the case of macroscopically rough surfaces, showing structures of more than $0.1 \mu \mathrm{m}$ in size, the tip usually is sufficiently sharp and does not infuence the realospace image of the surface's structures."

The major part of polycrystalline, (ion-) etched singlecrystalline or amorphous materials, however, exhibit surface features between these limiting values. Within this intermediate range of mesoscopically rough surfaces the tip shape clearly is decisive for the quality of STM images (tip-shape limited resolution). Two limits are obvious: (i) Tip radius $r$ is much smaller than typically size $R$ of the surface structures; here the STM image shows mainiy the real topography of the surface; (ii) $r \gg R$, i.e., the STM image reflects mainly the shape of the tip.

For many topographies, as for example the surfaces of polycrystalline thin films, imaged with conventionally manufactured tunneling tips, however, rather the case $r \approx R$ has to be considered. Therefore, the STM image represents a convolution between real topography and shape of the tip. This aspect of STM imaging of mesoscopically rough surfaces was already qualitatively discussed for example by Gimzewski et al. ${ }^{7}$ for polycrystalline Ag films, Niedermayer et al. ${ }^{8}$ for granular high- $T_{c}$ films and by Bartolomé et al. ${ }^{9}$ for STM images of an opticai disk. Quantitatively, however, the limits of resolution have not yet been treated, perhaps mainly owing to the lack of informations concerning the effective tip shape.

In ozder to investigate these limits, a proper estimation of the tip shape combined with a precise knowledge about the typical features of the surface therefore are necessary.

\section{TIP CHARACTERIZATION}

\section{A. Etching procedure}

The tips used for atomic resolution often exhibit overall tip radii between 0.2 and $0.5 \mu \mathrm{m}$, far too large for a reasonable resolution on rough surfaces. These tungsten tips are commonly etched at the interface between a $\mathrm{NaOH}$ or a $\mathrm{KOH}$ bath and air. More complicated etching procedures, however, have been shown ${ }^{8}$ to produce sharper tunneling tips.

In order to reduce the tip radius, we therefore employed a slightly modified procedure: A tungsten wire (diameter 0.5 $\mathrm{mm}$ ) is dipped into an inert liquid (trichlorethylene) covered by the etching solution ( $10 \% \mathrm{NaOH})$. The wire then is etched electrochemically $(\mathrm{ac}, 2-10 \mathrm{~V})$ at the trichlorethylen-NaOH interface using a carbon counterelectrode. This procedure gives needles ending into a wiskerlike tip of up to 5 $\mathrm{mm}$ length and down to $10 \mu \mathrm{m}$ diameter. This very end of the tip then is etched back to a length below about $1 \mathrm{~mm}$ in the common way at the interface between air and etching solution.

\section{Estimation of the tip shape}

In Fig. 1, a typical scanning electron microscopy (SEM) image of our tips is presented. The moderate magnification shows a tip ending into a small needle of about 0.5 mm in length and suggests an exponential shape at the end. Whereas the radius of curvature at the end of the tip $(r)$ cannot be estimated from this image, the cone angle $\phi$ is well below $10^{\circ}$ over the length of this needle. Larger SEM magnifications up to $2 \times 10^{4}$, however, demonstrate that the sign of the curvature changes about $3-4 \mu \mathrm{m}$ before the end of the tip is reached; the shape at the end thus is rather parabolic. The cone angle again can be estimated to be smaller than $10^{\circ}$ over a length of several $\mu \mathrm{m}$. This very small angle, however, must not be identified with the real cone angle at the very end of the tip, because the radius of curvature $(r)$ at the front of the tip turns out to be smaller than the resolution of our SEM $(50 \mathrm{~nm})$. 


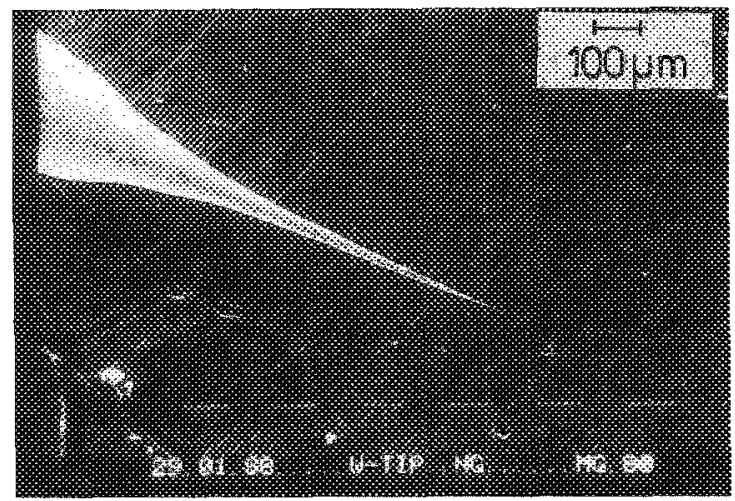

FIG. 1. A typical scaming electron microscope image of our tunneling tips.

A further rough test of the radius can be supplied by measuring the onset voltage of field emission of the tip. Whereas a commercial fiel cmission tip of $0.1 \mu \mathrm{m}$ radius shows onset at about $2 \mathrm{kV}$, our tips give field emission currents already at voltages down to $250 \mathrm{~V}$, using the same experimental arrangement.

Summarizing the informations obtained from SEM images and field emission measurements, the upper limit of the tip radius therefore is far below $50 \mathrm{~nm}$, whereas the cone angle is larger than about $10^{\circ}$.

For this information is not sufficient for an estimation of the resolution produced by such tips, STM itself has been used to image the tip shape: As shown in a previous publication, ${ }^{6}$ cleaved single-crystalline $\mathrm{NaCl}$ shows large cleavage steps; large and steep single steps up to about $80 \mathrm{~nm}$ in height can be found by STM on this (Au-covered) surface. If the tip crosses the sharp edge of a cleavage step, the image clearly does not show this cornered form but will give an upside down picture of half the tip itself (corresponding to $r \gg R$ ). If the upper edge of the cleavage step is not perfectly, i.e., atomicaly, sharp, this image of the tip will be slightly smeared out. The evaluation of the shape using such pictures therefore can provide much better upper estimates of the effective shape of the tip.

Figure 2 shows the result of turning the image of a $\mathrm{NaCl}$ cleavage step (height $80 \mathrm{~nm}$, see Ref. 10) upside down and doubling the result by refecting it at the plane located at

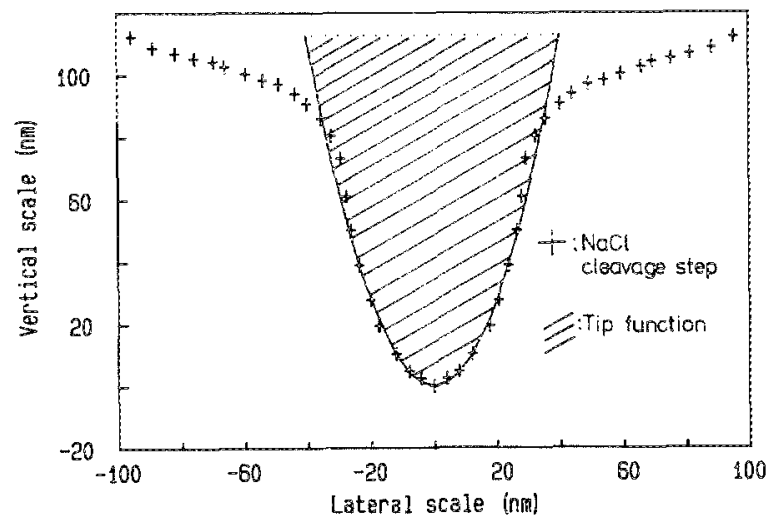

FIG. 2. Upside down single line scan of a NaCl-cleavage step reflected at $x=0(+)$ and fitced tip-shape (line). $x=0 \mathrm{~nm}$ (i.e., the location, where the first sign of the step can be recognized in the original picture). The result (crosses in Fig. 2) gives a rather exact estimate of the shape of the tip: The overall form is parabolic; the radius of curvature at the front amounts to about $(7.5 \pm 3) \mathrm{nm}$. The cone angle can be evaluated to be about $30^{\circ}$ over a length of $80 \mathrm{~nm}$; this is considerably larger than the macroscopic cone angle resolved by SEM, Fig. 1 .

This experimental shape can be fitted very well by the relation

$$
\operatorname{tip}(x)=\alpha x^{2} /(1+\beta|x|)
$$

using $\alpha=(0.07 \pm 0.03) \mathrm{nm}^{-1}$ and $\beta=(0.01 \pm 0.005)$ $\mathrm{nm}^{-1}$ (hine and shaded area in Fig. 2). The above function deseribes a tip with radius of curvature of about $1 / 2 \alpha$ and cone angle $\varphi$ of $(\pi / 2)-\arctan (\alpha / \beta)$.

One notices that these characteristics of our tunneling tips are considerably different from the estimation of Gimzewski et $a l^{7}$ for their tungsten tips $(r<1 \mathrm{~nm})$. These authors, however, estimated the tip radius from the lateral resolution of the tunneling graphs using the theoretical relation proposed by Stoll ${ }^{1}$ and Tersof and Hamann, ${ }^{2}$ which was developed for atomic resolution on microscopically flat surfaces.

In contrast with their method, we estimated directly the tip shape from $S T M$ scans at a sharp edge $(r \gg R)$. The evaluation of the effective tip shape allows for a quantitative discussion of the tip-limited resolution of STM images of mesoscopic surfaces.

\section{TIP-LMITED RESOLUTION: COMPARISOM OF STM AND TEM}

According to the foregoing discussion, the surface ideal for this purpose should exhibit mesoscopic structures $(r \approx R)$, i.e., between 5 and $50 \mathrm{~nm}$. Au-Nb-Au triple layered thin films on $\mathrm{Si}$ ( 100 ) substrates turned out to be very proper for this purpose. The experiment was performed in three steps: The fim production by evaporation in moderate ultrahigh vacuum $\left(10^{-8}\right.$ mbar) was already described in Ref. 10. During each evaporation process, eight substrates have been covered under identical surrounding conditions.

The surfaces of the films were then imaged by two different methods: one-half of the finms was transferred to a highresolution transmission electron microscope (TEM, Phillips CMI 30 ) and prepared for cross-section imaging by ion-milling. The rest of the substrates was used for STM imaging of the surfaces. This was done either by using complete substrates or by cutting them in two. In any case, the pictures obtained by both methods have been very reproducible ( see Ref. 10) and indicated a homogeneous film growth, i.e., the typical features are the same on different locations on the surfaces.

A typical TEM cross-section image of $A u_{12} \mathrm{~nm}-\mathrm{Nb}_{6 \mathrm{~nm}}$ $\mathrm{Au}_{12.5 \mathrm{~mm}}$ film is shown in Fig. 3: Whereas the first Au-layer grows smoothly on the Si substrate, the thin $\mathrm{Nb}$ layer gives rise to large mechanical stresses; therefore, the upper Au layer shows islandlike growth. Figure 3 shows smooth layers of the first ( $\mathrm{Au}$ ) and second ( $\mathrm{Nb}$ ) layer, whereas the upper (Au) fim exclusively consists of $15-30$ nm in height and $20-$ 


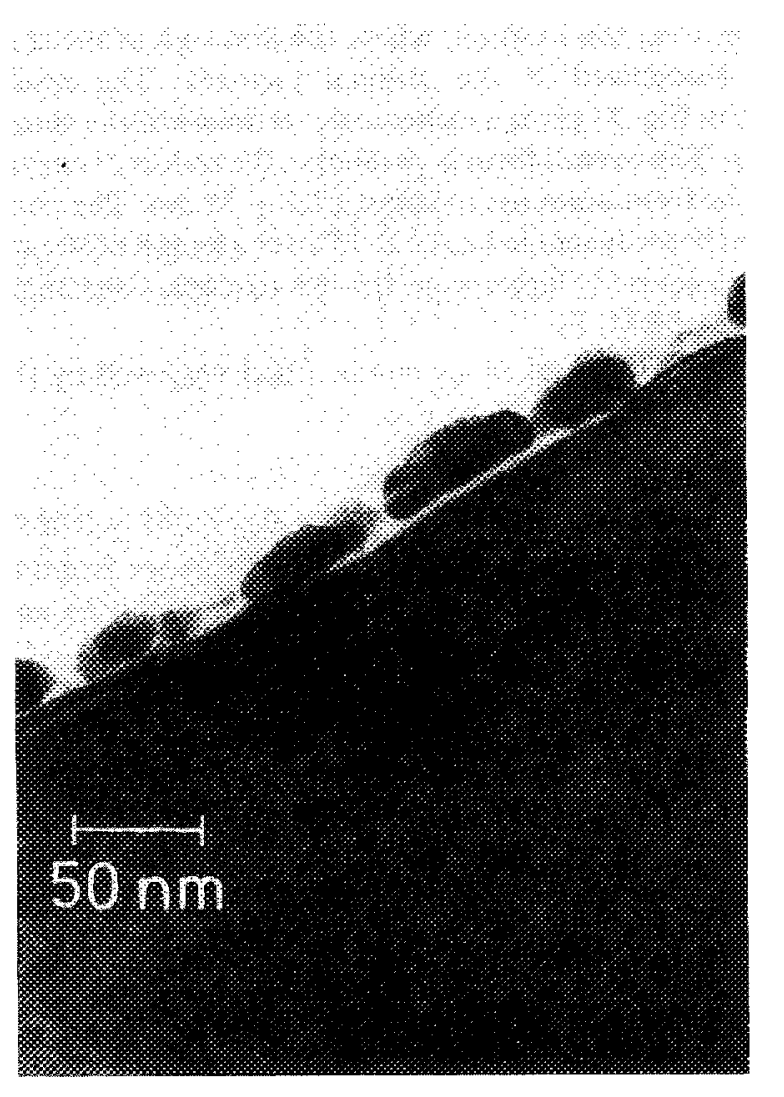

FIG. 3. TEM cross-section image of a $A u_{12} \mathrm{~nm}-\mathrm{Nb}_{6 \mathrm{am}}-\mathrm{Au}_{12.5 \mathrm{~nm}}$ triple layer. The islands on the top are formed by the upper Au layer.

$50 \mathrm{~nm}$ in width, separated by distances of about $10-30 \mathrm{~nm}$. These films therefore show surfaces very useful for an estimation of the tip-shape-limited resolution of the STM.

A typical STM image of a corresponding sample is shown in Fig. 4: Whereas the TEM cross section only reveals a islandlike structure, the STM image does not show compietely isolated Au-islands. Large hills of $15-25 \mathrm{~nm}$ in height (i.e., larger than the thickness of the Au coverlayer) and 20 $40 \mathrm{~nm}$ in width clearly point to island structure where smaller features seem to form a polycrystalline but continuous surface.

Owing to the knowledge of the tip shape, the images of TEM and STM can be quantitatively compared: For this

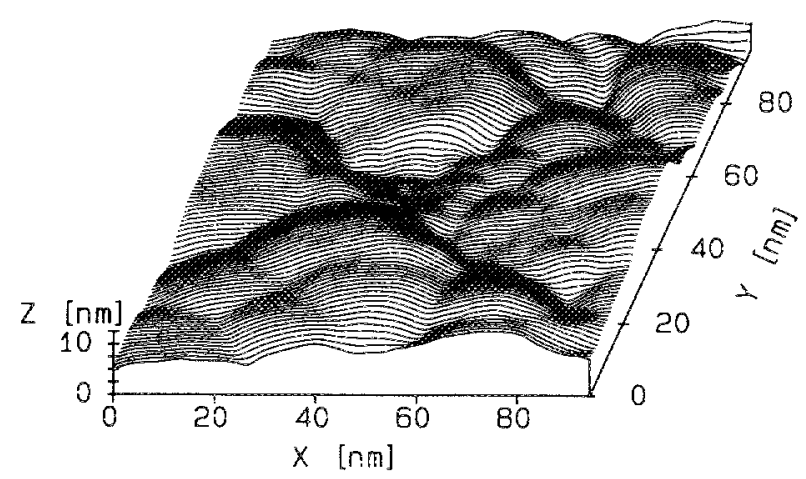

FIG. 4. STM inage of the suriace of a $A u_{12} \mathrm{~nm}-\mathrm{Nb}_{6, \mathrm{~mm}}-\mathrm{Au}_{12.5}$ nim triple layer.

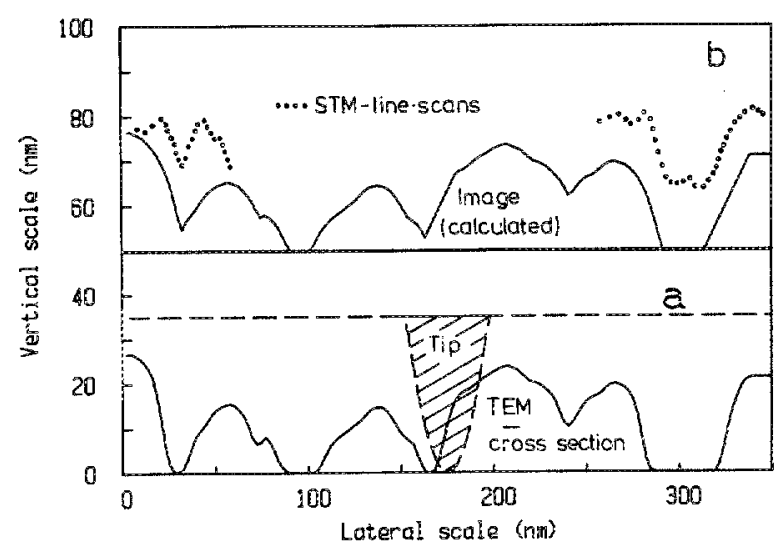

FIG. 5. (a) Digitized TEM cross section of a $A u_{12 . \pi m}-N h_{6 n}-A_{2} u_{12.5} \mathrm{~nm}$ triple layer and shape of the tunneling tip. (b) Convolution of TEM cross section and tip shape (solid line). Dotted curves: Line scans taken from the complete STM image (Fig. 4 ).

purpose the cross-section TEM image of Fig. 3 was digitized and numerically convoluted with the tip function of Fig. 2, assuming an STM working in constant current mode. The result is presented in Figs. $5(a)$ and $5(b) \quad(\alpha=0.07$, $\beta=0.01$ ); Fig. 5(a) shows the digitized TEM curve and the shape of the tunneling tip. Note that the tunneling distance is much smaller than the topographic features discussed here. ${ }^{12}$ The resulting calculated single-line STM scan [Fig. $5(b)$, solid line $]$ is compared with typical measured topographical features taken from the complete STM picture [dotted curves in Fig. 5(b)]. A good correspondence of calculated and measured features can be recognized: Whereas large structures are well resolved [right curves in Fig. 5(b)], smaller holes of the real surface appear only as triangular hollows in the STM graph (left curve). This calculation demonstrates, that only large islands (i.e., those with $R>r$ ) can be resolved by the tunneling tip. Smaller structures (i.e., $R<r)$, however, are smeared out and therefore the calculated STM image does not show the completely islandized structure of the TEM cross-section picture. Note that these typical triangular hollows are often interpreted as grain boundaries; concerning the location this may be correct for continuous polycrystalline fims. Due to the limits of resoluion demonstrated by Fig. 5, however, these interpretations have to be taken with care especially concerning the structure of these boundaries.

Therefore, although the TEM cross section clearly revealed a complete island structure of the surface, only a (hypothetic) tip with $r<3 \mathrm{~nm}$ would clearly resolve all Auislands. For a tip radius considerably larger than this value, reliable information can be obtained only about the lateral extensions of the surface's structures, whereas vertical ones will be strongly smeared out (Fig. 5).

Doing STM on such surfaces with regard to the results discussed above, an overall criterion for the tip-shape-limited resolution should be taken in mind: STM images of rough surfaces always represent a convolution of real surface structure and shape of the tip, which may change from one experiment to the other. Whereas the lateral extension of the surface's structures will be slightly enlarged, but reproduced 
with reasonable accuracy, the vertical ones will be strongly supressed by the effective shape of the tip. Rectanguiar holes of width $H$ and depth $T$, for instance, will be resolved only as long as the inequality

$$
\left(\frac{H}{2}-r\right) \tan \left(\frac{\pi}{2}-\varphi\right)+r>T
$$

holds. Otherwise this hole will be smeared out and reproduced only by a triangular hollow with width $H$ but considerably reduced depth. If, in turn, hills of lateral size $R$ and height $H$ are completely separated by such holes, the real structure can be recognized only as long as this condition remains valid, alhough the lateral size shown by STM images would be only slightly enhanced (below or equal to about $R+2 r$ ). This inequality therefore approximately holds for our islandlike structured thin fims, too.

In contrast with imaging micrsocopically flat or macroscopically rough surfaces, the results of STM measirements on mesoscopically structured surfaces therefore must be interpreted always including the specific geometric shape of the tip and be compared with the results of other methods capable of mesoscopic resolution. Efforts concerning the de- convolution of STM images with typical tip shapes are in progress.

We gratefully acknowledge the help of F. Schneider for the STM measurements.

1. Tersoff and D. R. Hamann, Phys. Rev. Lett. $\$ 0,1998$ (1983).

2J. Tersoff and D. R. Hamann, Phys. Rev. B $\$ 1$, 805 (1985)

${ }^{3}$ J. Tersof, Phys. Rev. Lett. 57, 440 (1986).

${ }^{4}$ H. A. Mizes, S. Park, and W. A. Harrison, Phys. Kev. B 36. 4491 (1987).

5R. J. Cotton, S. M. Baker, R. J. Driscoll, M. G. Youngquist, and J. D. Bahdeschwieler, J. Vac. Sci. Techmol. A 6, 349 (1988).

'S. Okayama, M. Konuro, W. Muzutani, H. Tokunoto, M. Okano, K. Shimizen, Y. Kobayashi, F. Matsumoto, S. Wakiyama, M. Shigeno, F. Sakai, S. Fujiwara, O. Kitamura, M. Ono, and K. Kajimura, J. Vac. Sci. Technol. A 6,440 (1988).

7J. K. Gimzewski, A. Humbert, J. G. Bednorz, and B. Reihl, Bhys. Rev. Lett. \$5, $951(1985)$.

${ }^{8}$ Ph. Niedermann and Q. Fischer, 3 . Microse. $\mathbf{5 2}, 93$ (1988).

${ }^{9}$ A. Bartolomé, R. Garcia, L. Vázquez, and A. M. Baró, J. Microsc. 152, 205 (1988).

J. Vancea, G. Reiss, F. Schneider, K. Barer, and H. Hofmann, Surf. Sci. 218, 108 (1989).

"E. Stoll, Surf. Sci. 143, C143 (1984).

${ }^{12}$ N. D. Lang, Phys. Rev. B 37, 10395 (1988). 